\title{
ZDC Effective Cross Section for Run 12 Uranium-Uranium Collisions in RHIC
}

\author{
A. Drees
}

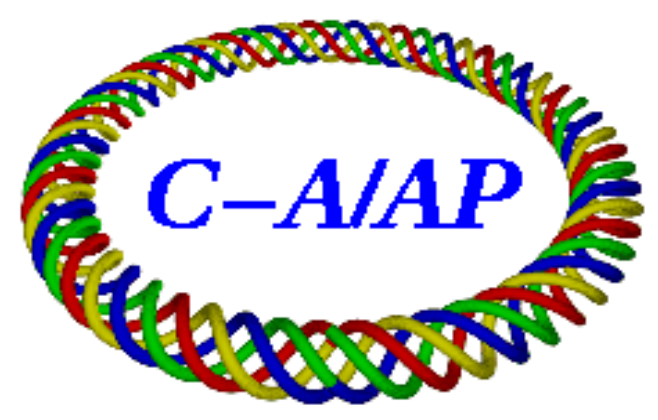

\section{Collider-Accelerator Department Brookhaven National Laboratory Upton, NY 11973}

Notice: This document has been authorized by employees of Brookhaven Science Associates, LLC under Contract No. DE-AC02-98CH10886 with the U.S. Department of Energy. The United States Government retains a nonexclusive, paid-up, irrevocable, world-wide license to publish or reproduce the published form of this document, or allow others to do so, for United States Government purposes. 


\title{
ZDC Effective Cross Section for Run 12 Uranium-Uranium Collisions in RHIC
}

\author{
A. Drees
}

December 23, 2013

\section{Introduction}

An accurate calibration of the luminosity measurement of the 2012 Uranium-Uranium RHIC run at $96 \mathrm{GeV}$ per beam is of the greatest importance in order to measure the total uranium-uranium cross section with a reasonably small error bar. During the run, which lasted from April 20th to May 15th 2012, three vernier scans per experiment were performed. Beam intensities of up to $3.410^{10}$ Uranium ions in one ring were successfully accelerated to flattop at $\gamma=103.48$ corresponding to $96 \mathrm{GeV} /$ beam. The desired model $\beta^{*}$ value was $0.7 \mathrm{~m}$ in the two low beta Interaction Points IP6 and IP8. With these beam parameters interaction rates of up to $15 \mathrm{kHz}$ were achieved. This note presents the data associated with the vernier scans, and discusses the results and systematic effects.

\section{Vernier Scan Technique}

During a vernier scan, also known as a Van der Meer scan [1], the transverse size and shape of the beam overlap region is measured by recording the interaction rate as a function of the transverse beam separation. A Gauss-fit of the measured interaction rate as a function of the separation allows to determine the effective beam size as well as the maximum achievable collision rate and thus the effective cross section of the detector in use. Typically additional effects such as the presence of debunched beam, a possible crossing angle or the hourglass effect [2] require correction factors to be applied to the results. In the case of the stochastically cooled UU beams [3], a simple Gauss-fit was not sufficient to describe the shape of the overlap region and a 2-Gauss fit had to be used to describe the data. The transverse beam size was derived by using a weighed average of the widths of two Gauss-functions. Table 1 lists all available vernier scans, the time the data was taken, the IP, the fill pattern and which beam was moved during the scan.

\subsection{The Fit Function}

The shape of the overlap region, i.e. the collision rate $R_{\text {coll }}(x)$ as a function of distance between the two beams, $x$, is mapped by the vernier scan and can usually best be described by a single Gauss-function:

$$
R_{\text {coll }}(x)=R_{\mathrm{Bkgd}}+R_{\max } \times \exp \left(\frac{-\left(x-x_{0}\right)^{2}}{2 \sigma_{\mathrm{x}}^{\mathrm{VS}}}\right)
$$

with the 4 free parameters:

$R_{\mathrm{Bkgd}}$ : non-collision related background signal in the collision rate ("offset") 


\begin{tabular}{|l|l|c|c|l|c|c|}
\hline fill & date & time & ring & IP & fill pattern & $n_{\text {coll }}$ \\
\hline \hline 16783 & $04 / 25$ & $19: 31-19: 49$ & B & IP6 & $111 \times 111$ & 102 \\
\hline 16783 & $04 / 25$ & $19: 54-20: 13$ & B & IP8 & $111 \times 111$ & 111 \\
\hline 16842 & $05 / 09$ & $12: 34-12: 53$ & Y & IP6 & $111 \times 111$ & 102 \\
\hline 16842 & $05 / 09$ & $12: 55-13: 11$ & Y & IP8 & $111 \times 111$ & 111 \\
\hline 16857 & $05 / 14$ & $15: 21-15: 34$ & Y & IP6 & $111 \times 111$ & 102 \\
\hline 16857 & $05 / 14$ & $16: 08-16: 20$ & Y & IP8 & $111 \times 111$ & 111 \\
\hline
\end{tabular}

Table 1: List of fills with vernier scans during the $2012 \mathrm{UU}$ run at $96 \mathrm{GeV} /$ beam.

$R_{\max }$ : maximum collision rate seen by the ZDC detector (corrected for background) $x_{0}$ : location of the maximum $\sigma_{x}$ : width of the overlap region

This approach is used when fitting data from a store with uncooled Uranium beams (such as store 16783). Fig. 1 shows the horizontal data and 1-Gauss fit to the STAR ZDC data during the 16783 vernier scan.

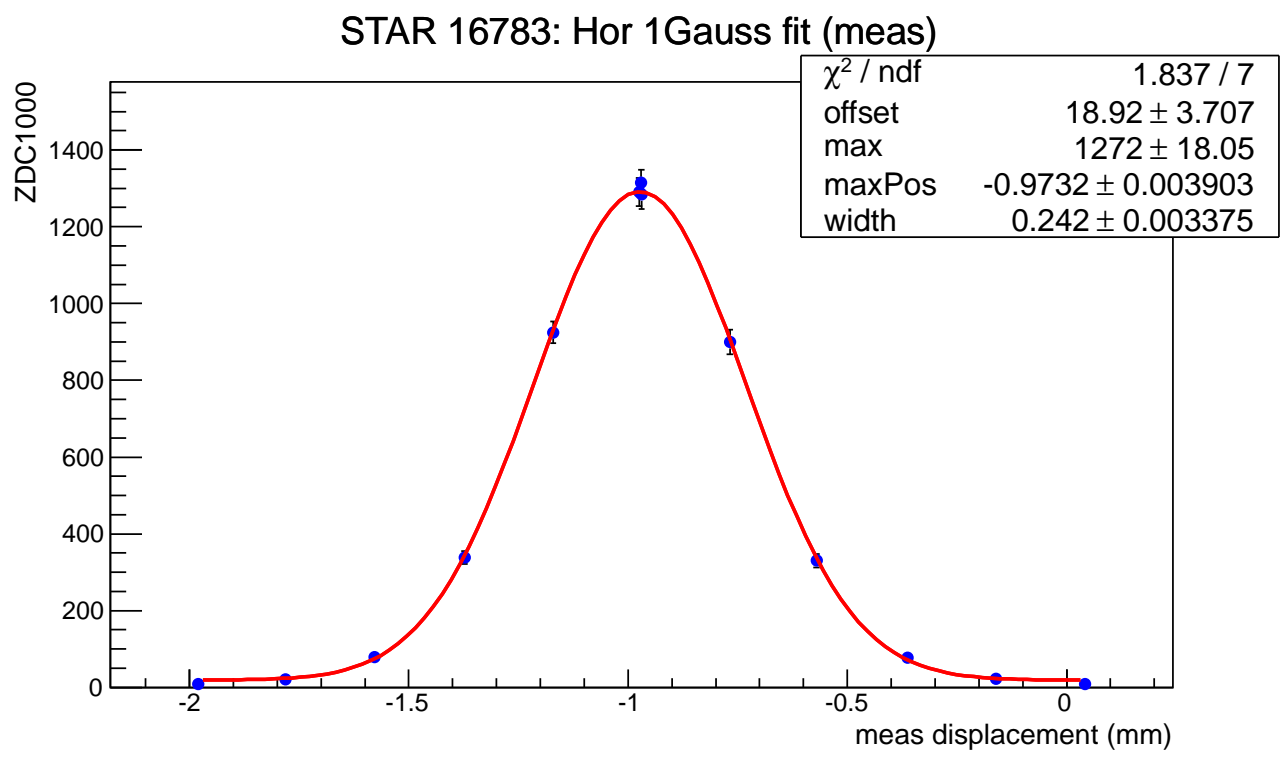

Figure 1: Horizontal data from a vernier scan in fill 16783 at the STAR experiment fitted with a 1-Gauss fit function. Stochastic cooling was not used.

However, in the case of stochastically cooled Uranium beams the single Gauss approach does no longer fit the data and a double Gauss-function is chosen:

$$
R_{\text {coll }}(x)=R_{\mathrm{Bkgd}}+R_{\max , 1} \times \exp \left(\frac{-\left(x-x_{0}\right)^{2}}{2 \sigma_{\mathrm{x}, 1}^{\mathrm{VS}}}\right)+R_{\max , 2} \times \exp \left(\frac{-\left(x-x_{0}\right)^{2}}{2 \sigma_{\mathrm{x}, 2}^{\mathrm{VS}}}\right)
$$

The double Gauss function has 6 free parameters:

$R_{\max , 1}$ : maximum collision rate of the core region 
$x_{0}$ : location of both the maxima

$\sigma_{\mathrm{x}, 1}$ : width of the core overlap region

$R_{\max , 2}$ : maximum of the tail distribution

$\sigma_{\mathrm{x}, 2}$ : width of the tail region

$R_{\text {Bkgd }}$ : non-collisional background rate ("constant")

The double Gauss approach was first necessary during the pp run at $250 \mathrm{GeV}$ in Run9 [4]. In this analysis it is assumed that both Gauss distributions, core and tail, are centered around the same location $x_{0}$. Data from stores 16842 and 16857 were fitted with the double Gauss. Fig. 2 shows the vertical PHENIX ZDC data together with a 2-Gauss

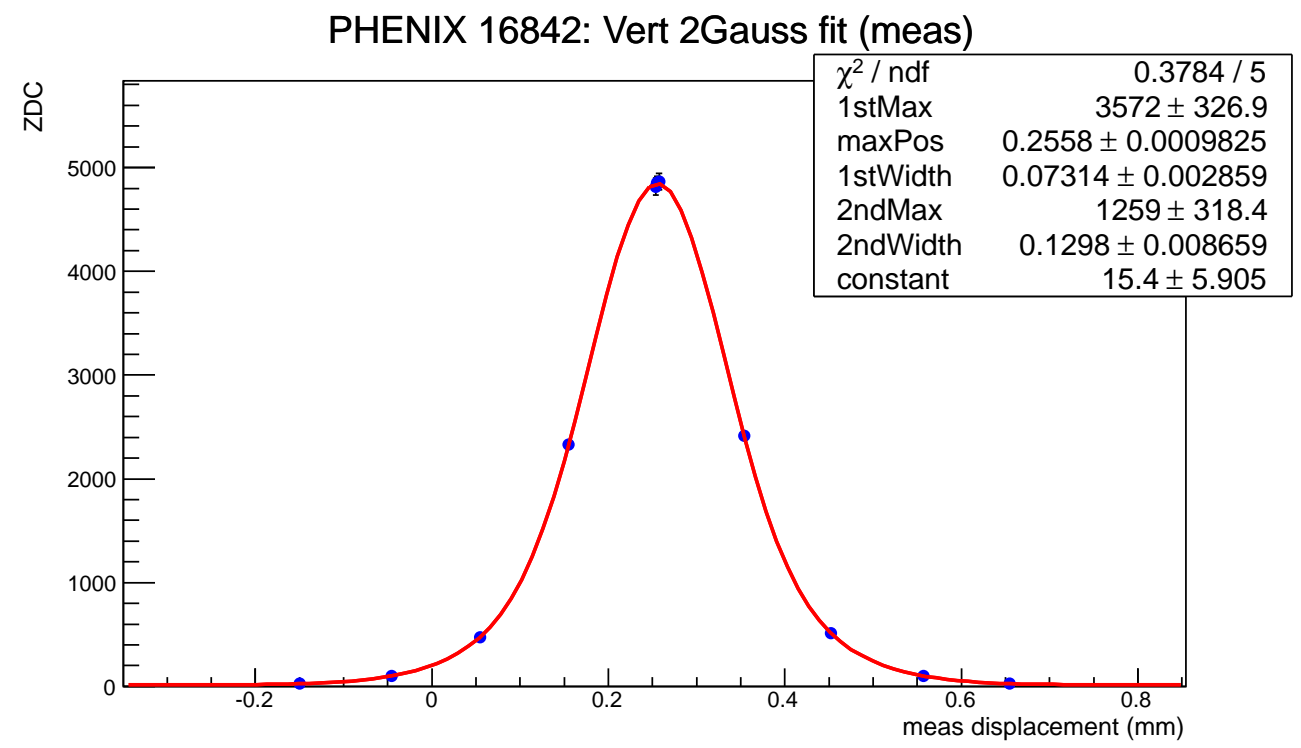

Figure 2: Vertical data from a vernier scan in fill 16842 at the PHENIX experiment fitted with a 2-Gauss fit function. Stochastic cooling was used during this store.

fit during the 16842 vernier scan. The beams were cooled during this store.

\section{The Effective Cross Section}

The effective detector cross section $\sigma_{\mathrm{ZDC}}^{\mathrm{eff}}$ of a detector, in this case the ZDCs, can be determined by the beam current, the collision rate and the overlap region $\sigma_{\mathrm{x}, \mathrm{y}}^{\mathrm{VS}}$ of the two beams. The maximum collision rate and the width of the overlap region are derived from the fits to the vernier scan data.

$$
\sigma_{\mathrm{ZDC}}^{\mathrm{eff}}=\frac{R_{\max } 2 \pi n_{B} n_{Y} \sigma_{\mathrm{x}}^{\mathrm{VS}} \sigma_{y}^{\mathrm{VS}}}{n_{\text {coll }} f_{\text {rev }} N_{B} N_{Y}}
$$

where:

$R_{\max }=$ maximum collision rate seen by the ZDC detector (corrected for background)

$n_{B}, n_{Y}=$ number of blue and yellow bunches respectively

$\sigma_{\mathrm{x}, \mathrm{y}}^{\mathrm{VS}}=\mathrm{RMS}$ beam-overlap size, derived from the fit to the vernier scan data

$n_{\text {coll }}=$ number of colliding bunch pairs in the IP where the ZDC detector is located $f_{\text {rev }}=$ revolution frequency, approx. $78.4 \mathrm{kHz}$ 
$N_{\mathrm{B}, \mathrm{Y}}=$ total number of ions in the blue and yellow rings, from WCM

Fig. 2 shows one example of a vernier scan, here in the vertical plane in IP8, moving the yellow beam. It depicts the ZDC coincidence rate as a function of the measured distance of the two beams. The data is fitted with a double Gauss function according to Eq. 2 . The beam overlap size $\sigma^{\mathrm{VS}}$ in Eq. 3 is given by the combination of the two widths from the double Gauss fit. The combined width adds the two individual widths according to their amplitudes:

$$
\sigma_{\mathrm{x}, \mathrm{y}}^{\mathrm{comb}}=\sigma_{1}^{\mathrm{x}, \mathrm{y}} \frac{R_{\max , 1}}{R_{\max , 1}+R_{\max , 2}}+\sigma_{2}^{\mathrm{x}, \mathrm{y}} \frac{R_{\max , 2}}{R_{\max , 1}+R_{\max , 2}}
$$

"x" and "y" refer to the two planes, while "1" and "2" refer to the core (1) and tail (2) part of the distribution respectively.

Several corrections apply to the cross section measurement using the vernier scan method. They potentially affect the collision rate (crossing angles, accidental coincidences, background), the beam current measurement (fill pattern and debunched beam) and the measurement of the width (beam-beam, beam separation measured by BPMs). The various effects are discussed below with the exception of accidental coincidences (see [6] for details) and backgrounds. Non-collisional backgrounds are accounted for in the fitfunctions and systematic errors due to accidental coincidences are negligible with collision rates of just a few $10 \mathrm{kHz}$. Regardless, the collision rates in the following are corrected for accidental coincidences.

\section{Corrections and Systematic Errors}

\subsection{Beam Current Measurement}

Due to Intra Beam scattering (IBS [7]) with heavy ions, a standard heavy ion store of a typical length of about 6 to 7 hours is subject to significant debunching of the beam. "Standard" in this context refers to uncooled beams. However, during the most part of the UU run in 2012 the heavy ions were not only stochastically cooled in the longitudinal but also the two transverse planes [3]. If debunched beam, or beam which does not contribute to collisions, is present in RHIC uranium-uranium stores, this analysis cannot rely on the measurement of the total circulating beam current. Thus it is mandatory to find proof for the presence or absence of debunched beam before we decide on which device to rely on for beam current measurement: the DCCT [8] or the Wall Current Monitor (WCM [8]). While the DCCT measures the total current circulating in RHIC, the WCM is sensitive to only the bunched portion of it. The differences between the DCCT and WCM include possible true debunched beam, a store to store variation of the WCM calibration to the DCCT and changes in the WCM readings due to gain changes in the scopes. These features are discussed below.

\subsubsection{Calibrating the WCM}

Two scopes are used as the data acquisition system for the WCMs. While an excess of bunched beam over total circulating beam has to be attributed to a calibration issue, it is of course possible that the amount of bunched beam is less than the total circulating beam. However, this does not apply to the period of time when RHIC is ramping when accelerated beam has to be bunched (or get lost). Therefore the WCM can be calibrated 
with the DCCT at the end of the ramp. For this analysis, ev-flattop was chosen to signify the end of the ramp. Tab. 2 shows the difference DCCT-WCM for the three vernier scan stores at "flattop". A negative sign signals that the WCM reading is above the DCCT

\begin{tabular}{|l|c|c|c|c|}
\hline fill & Blue [ions $\times 10^{9}$ ] & Blue [\%] & Yellow [ions x 10 ${ }^{9}$ ] & Yellow [\%] \\
\hline 16783 & -0.33 & -1.25 & -0.4 & -1.54 \\
\hline 16842 & -0.39 & -1.25 & -0.46 & -1.53 \\
\hline 16857 & -0.38 & -1.17 & +0.22 & +0.64 \\
\hline
\end{tabular}

Table 2: Blue and yellow WCM calibration for the three vernier scan stores in units of ions $x 10^{9}$ and percent of total beam respectively.

reading at the time the ramp reaches "flattop". Looking over a random selection of other UU stores revealed large store to store variations and a needed correction of the WCM readings of up to $2 \%$. However, the values found in the first two vernier scan stores appear to be rather typical. Due to the availability of the DCCT (with a factory listed absolute accuracy of $0.2 \%$ ) the varying calibration of the WCM could be corrected for with an associated systematic error of $2 \times 0.2 \%$.

In order to estimate the point-to-point measurement accuracy or scatter of the WCM readings, a data set from store 16857 corresponding to about $1 \mathrm{~h}$ of data taking was selected. During this one hour neither the blue nor the yellow scope did undergo a gain change and the beam current dropped linearly by approximately $10 \%$ in this time period. The linear drop was fitted and the fit subtracted from the data to compensate for ordinary beam loss in the data sample. The resulting two distributions are shown in Fig. 3. The
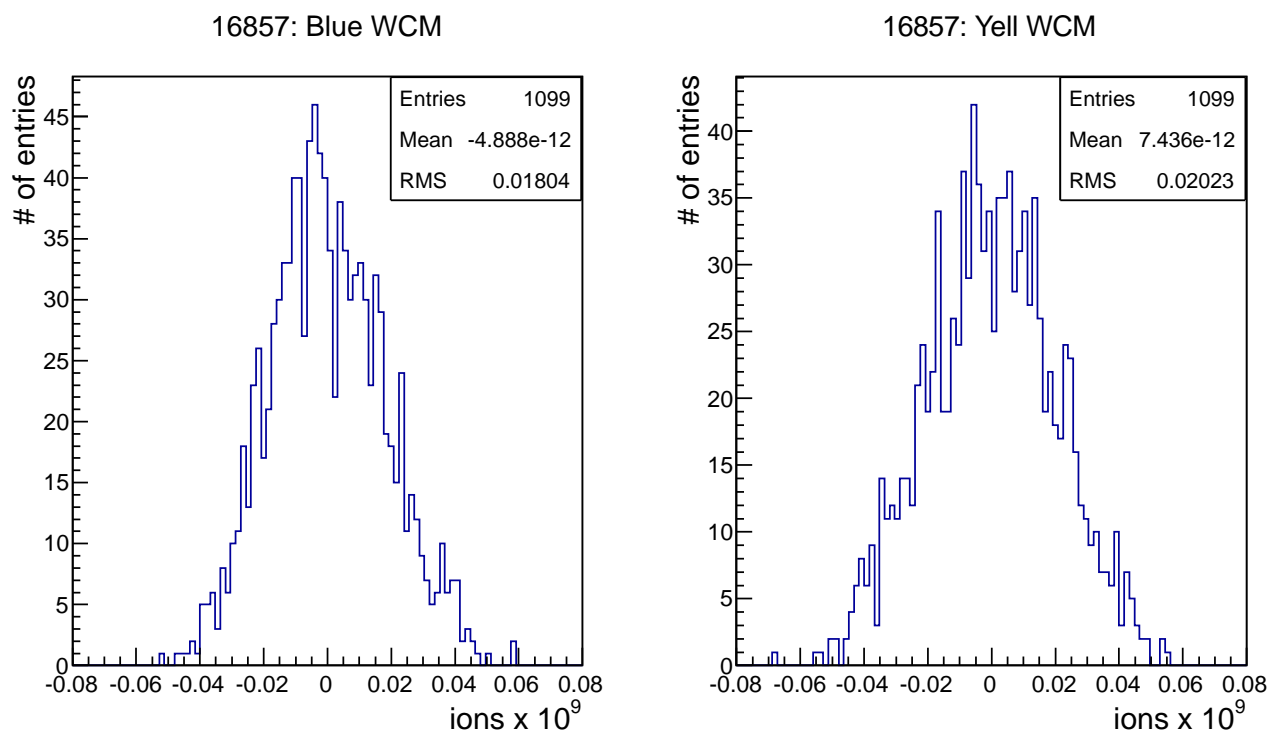

Figure 3: Point-to-point measurement scatter as seen by the blue (left) and yellow (right) WCM scope for the time period of about $1 \mathrm{~h}$ in store 16857.

RMS of the scatter, 0.02 ions $\times 10^{9}$, is identical for the two scopes corresponding to a $0.2 \%$ effect per ring given a minimum beam current of $12 \times 10^{9}$ ions at the end of some 
UU stores. Thus a combined systematic error of $2 \times 0.2 \%$ is assigned to cover the scatter in the WCM point-to-point measurements.

\subsubsection{Debunched Beam}

One indicator for the presence of true debunched beam is an apparent difference between the DCCT and WCM, especially if this difference is not constant throughout a store as would be the case with a simple calibration offset. Fig. 4 contains three graphs showing
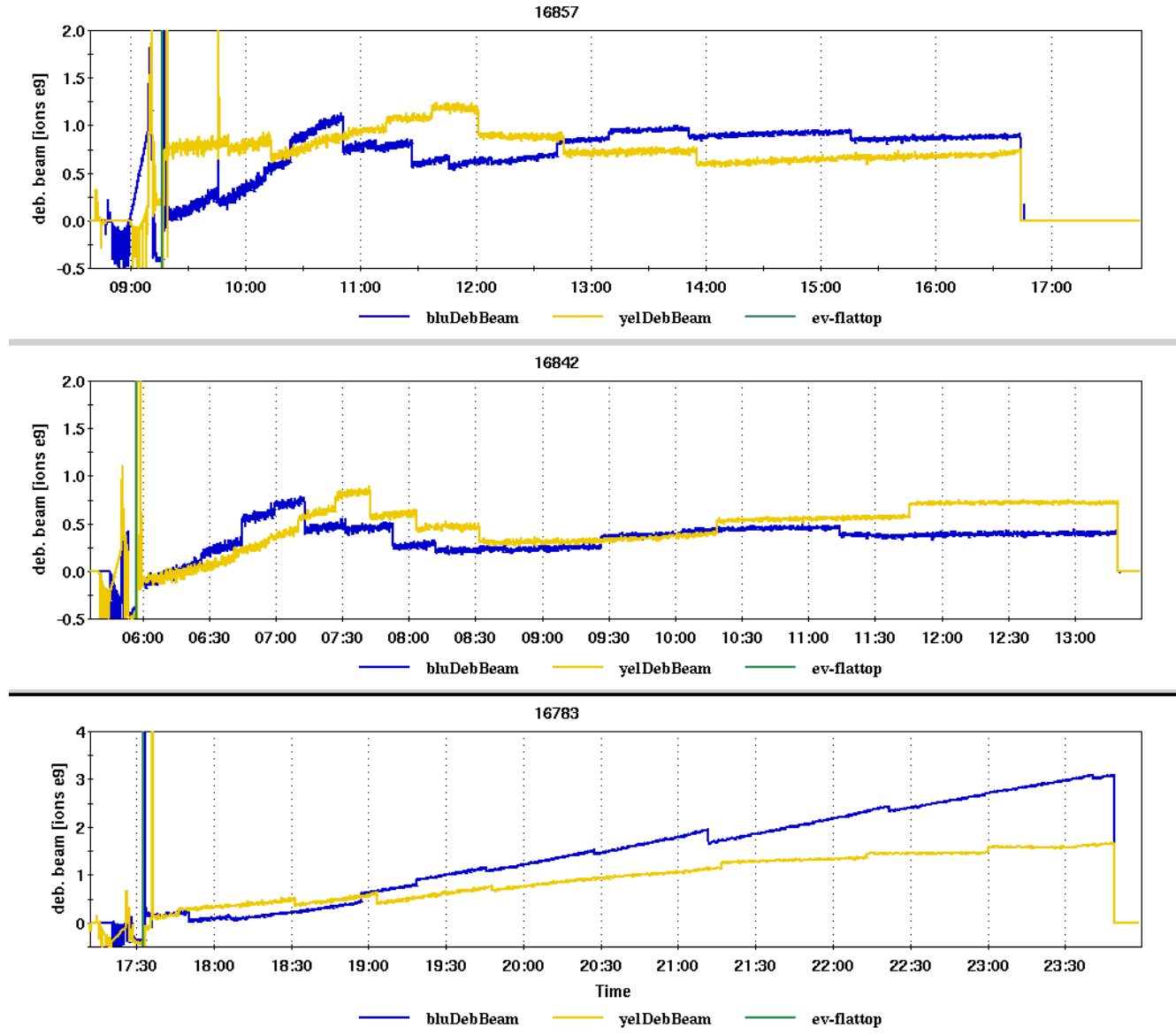

Figure 4: Difference of the DCCT and WCM readings as a function of time for the three stores with vernier scans. A positive reading or slope indicates an increase of debunched beam in RHIC, equivalent to a loss of bunched beam.

the difference between the two available devices, DCCT and WCM, in the two rings for the three vernier scan stores. Units are number of ions $\times 10^{9}$. The bottom graph, showing the store with the first vernier scan, depicts a steady and linear increase of the amount of debunched beam due to the absence of cooling. Clearly, the WCM has to be used to analyse data from this scan. The center and top graph, however, showing the two other stores with vernier scans, depict much less of an increase but nevertheless a steady upward slope within the first 1 to 2 hours of the store. The readings are stable after that (neglecting the effect of gain changes for this discussion, see section 4.1.3). The time frame of 1 to 2 hours corresponds to about the time it took for the bunch length to reach the 
initial value again after a period of shortening and lengthening due to longitudinal cooling.

In addition to evidence of some ongoing debunching during the first hours of a store, there appears to be some loss of bunched beam associated with the rebucketing exercise. Rebucketing is performed as soon as the ramp is finished, within less than $100 \mathrm{sec}$ after reaching flattop. The loss of beam at rebucketing in the three vernier scan stores is summarized in Tab. 3. According to the WCM, some beam is lost in all three cases. Note that going through rebucketing is always accompanied by a gain change in the WCM scopes. Gain changes in the scopes are known to cause distinct changes in the WCM readings that are solely due to the change in the scope gain and not due to a true change of bunched beam. The effect of gain changes is discussed in more detail in section 4.1.3 below. Such distinct changes in the WCM readings (increase as well as decrease) can easily be seen in Fig. 4 above for all three stores.

\begin{tabular}{|l|c|c|c|c|}
\hline fill & Blue [ions x $10^{9}$ ] & Blue [\%] & Yellow [ions x 10 ${ }^{9}$ ] & Yellow [\%] \\
\hline 16783 & -0.530 & -1.97 & -0.306 & -1.19 \\
\hline 16842 & -0.344 & -1.15 & -0.372 & -1.23 \\
\hline 16857 & -0.405 & -1.17 & -0.484 & -1.45 \\
\hline
\end{tabular}

Table 3: Loss of bunched beam at rebucketing in units of ions $\mathrm{x} 10^{9}$ and percent of total beam as measured by the WCM.

16857 Yellow Scope (zoom)

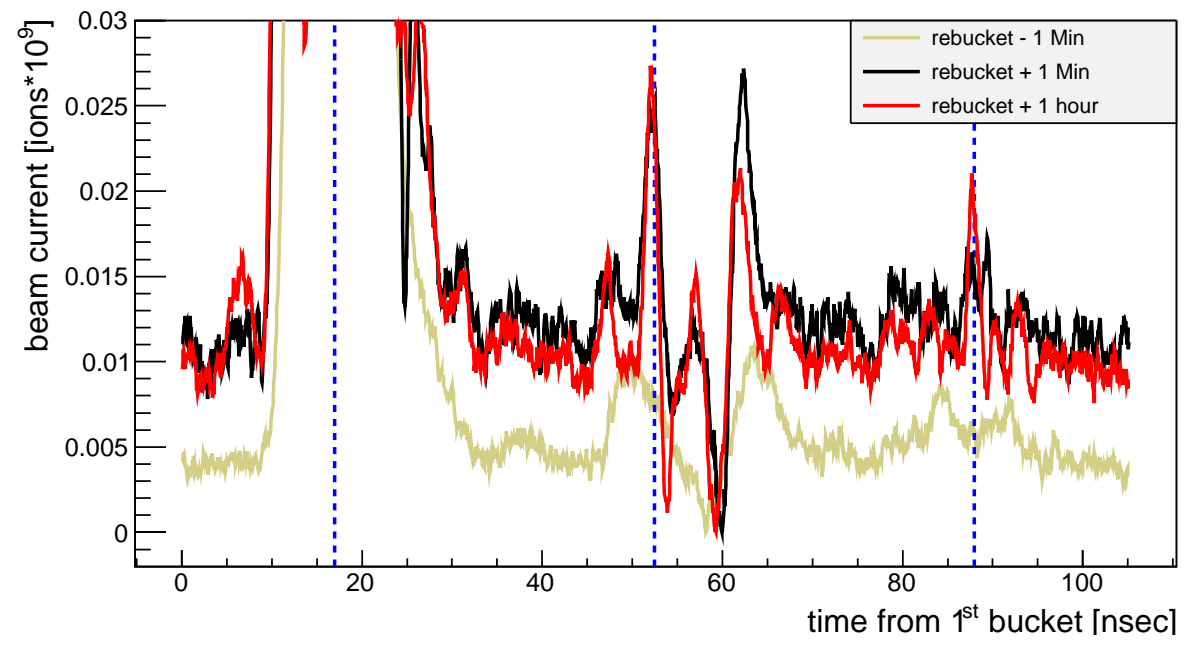

Figure 5: Three traces from the yellow WCM scope, each combining all 120 buckets in RHIC, taken at various times before and after rebucketing. Vertical scale is zoomed for better visibility.

The amount of beam loss at rebucketing is significant in all three cases in Tab. 3. If it is real, the beam could be visible in the WCM scope. Fig. 5 shows three scope traces from the yellow scope in store 16857. Each trace corresponds to an average of all 120 sets of consecutive 3 buckets [9] in RHIC where only the first of the 3 buckets is expected to be filled with beam. The vertical dashed blue lines correspond to the centers of the 3 
buckets. The first trace (tan colored), taken about 1 minute before rebucketing, shows no apparent signal besides the main bunch in bucket \#1 of the 3 shown buckets and some noise. The second trace (black) is taken 1 minute after rebucketing. Some beam appeared in the neighboring bucket \#2 together with a pattern (centered around $60 \mathrm{nsec}$ ) that can perhaps be attributed to ringing in the scope signal. The third trace (red) is taken 1 hour after rebucketing. Enough time passed for cooling to take effect and trap beam in any of the 3 buckets. A small but centered signal appeared in bucket \# 3 . This beam, while not debunched in the literal sense, will not contribute to collisions.

Due to the incontrovertible presence of some, however small, amounts of debunched beam in UU stores, the WCM will have to be used for this analysis in lieu of the more accurate DCCT.

\subsubsection{Gain Changes}

As stated above, the rebucketing procedure is always accompanied by a gain change in the WCM scopes. Since the WCM has to be used it is of interest how much of the rebucketing loss is real rather than a gain change artifact. In order to estimate which portion of the apparent beam loss is debunched beam and how much is due to a gain change, a total of $36 \mathrm{UU}$ stores were analyzed. In addition to the rebucketing gain change there are approximately 10 more asynchronous gain changes later during the store. Most of them cause the WCM reading to change distinctly. The WCM changes have both signs. For this analysis 7 stores were randomly selected in addition to the 3 stores with vernier scans yielding 108 blue scope gain changes at store and 93 yellow scope gain changes at store. The gain changes at rebucketing are not part of this set. Blue and yellow datasets were combined since both reveal the same general behavior. Fig. 6 contains histograms of both

\section{WCM after-before gain change}

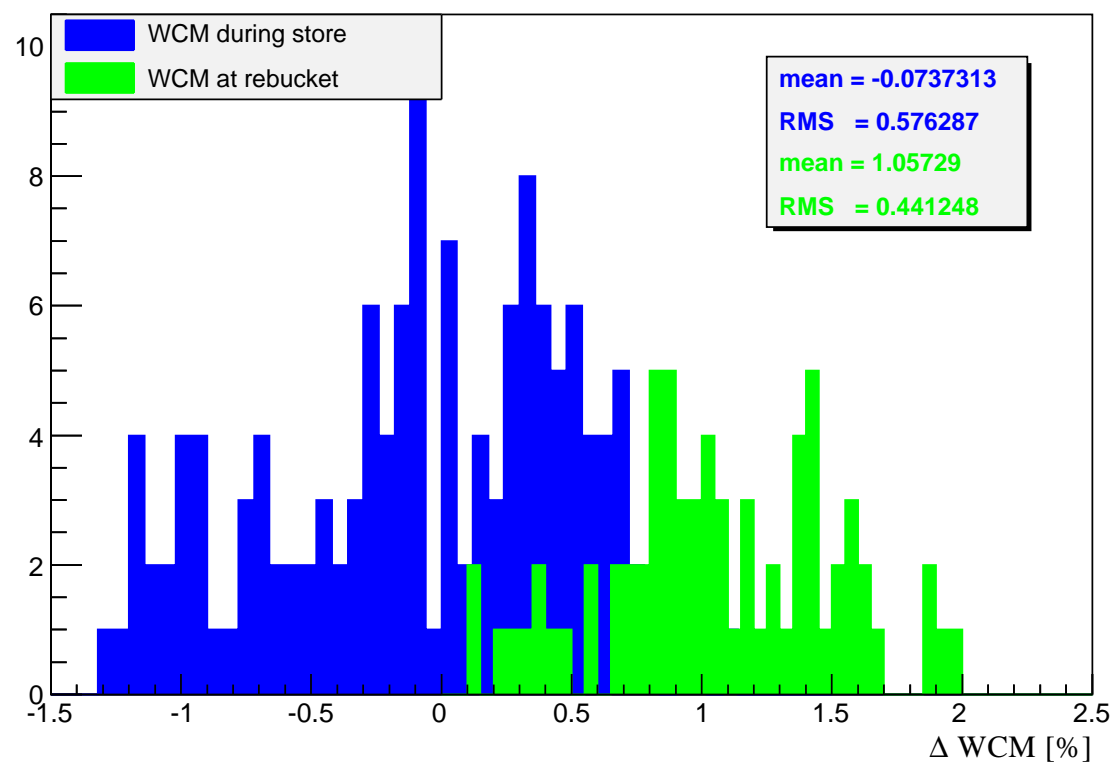

Figure 6: Histogram of two datasets containing scope gain changes (blue) and rebucketing changes (green). Blue and yellow data are combined. 
datasets, at store and at rebucketing. The blue shaded histogram shows the number of entries with distinct changes in the WCM readings caused by a gain change of the scope while at store. The amount of change is given in percent of beam current at the time and is calculated as the difference of the reading after and before the scope changed its gain. The mean and RMS value of this distribution are printed in blue inside the graph. The mean is consistent with 0 with an RMS of $0.58 \%$. The green shaded histogram shows the number of entries with the quoted amount of change in the WCM readback that is associated with rebucketing. The mean is shifted from 0 to $+1 \%$, with an RMS of $0.4 \%$.
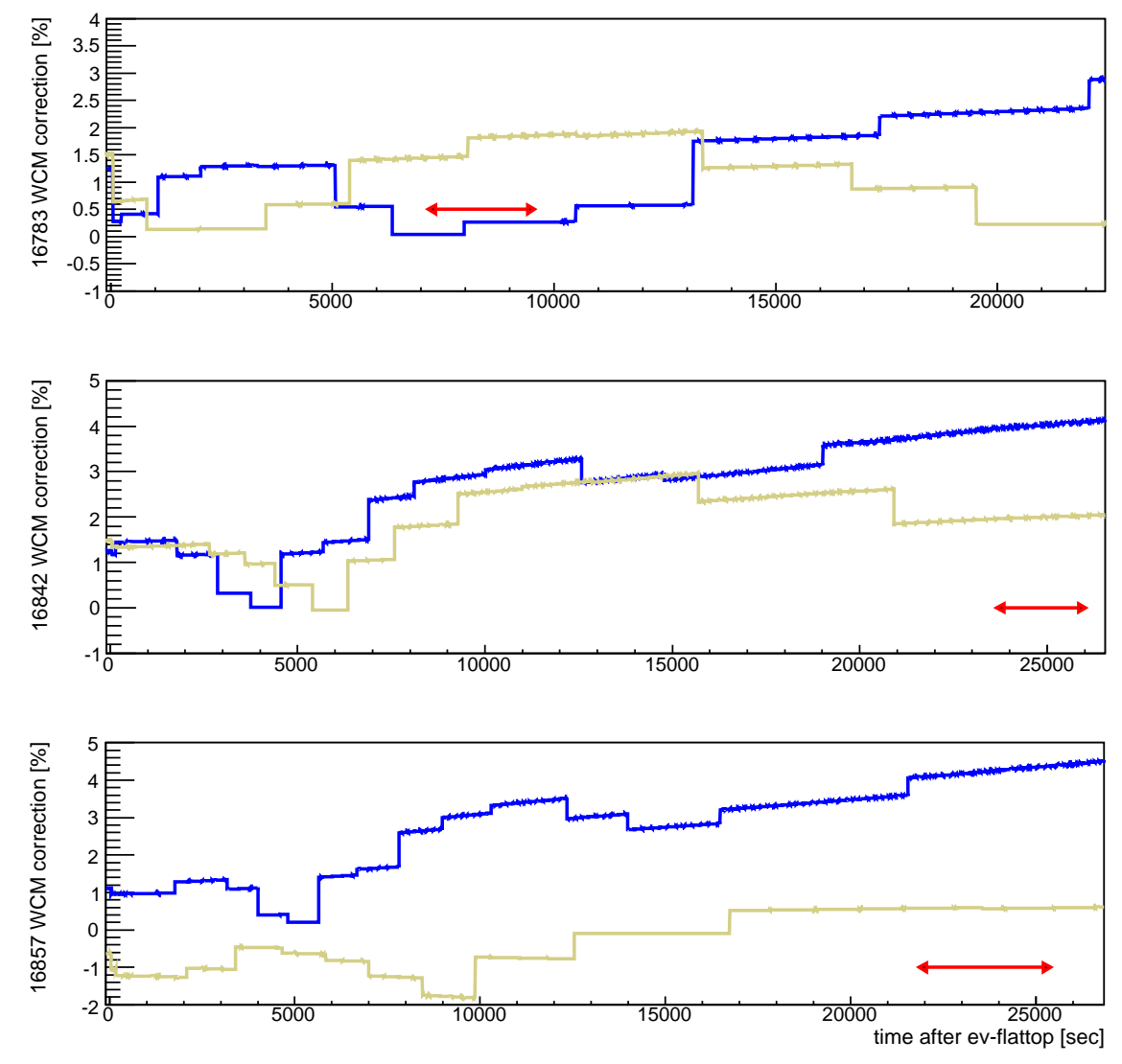

Figure 7: Size of the correction to the WCM readings for the blue and yellow rings at store as a function of time after reaching "flattop" for the three vernier scan stores.

This distribution is clearly not consistent with the other. While the $\triangle \mathrm{WCM}$ due to store gain changes scatters around 0 , this distribution shows a mean value that is not consistent with 0 but with an average bunched beam loss of $1 \%$. This signifies a true loss of bunched beam at rebucketing of an average of $1 \%$ of the beam current which is superimposed by a WCM reading shift triggered by the scope gain change. The extra fake shift causes the measured beam loss at rebucketing to be decreased or increased, depending on the random nature of the shifts caused by gain changes. Thus, for this analysis, the bunched beam current at rebucketing is reduced by $1 \%$ for each individual vernier scan store with an associated systematic error of $0.4 \%$ per beam totaling $0.8 \%$.

What is left to do is a correction for all following gain changes during the store based on the assumption that none of the associated WCM shifts are real. The first step is a 
correction of the WCM reading based on the calibration with the DCCT at the end of the ramp (see section 4.1 .1 above). This is followed by the $1 \%$ correction at rebucketing as described above. The effect of each following gain change is erased assuming that the calibrated reading from before the gain change is accurate. Fig. 7 shows the accumulating corrections in percent for the three vernier scan stores. The time ranges during which the vernier scans happened are indicated by the red arrows. The corrections to the blue and yellow WCM readings are shown as a blue and yellow line respectively. At the time of the vernier scan the corrections range from practically 0\% to up to 4\% (blue in 16842 and 16857). Fig. 8 shows the corrected and uncorrected beam current as measured by the
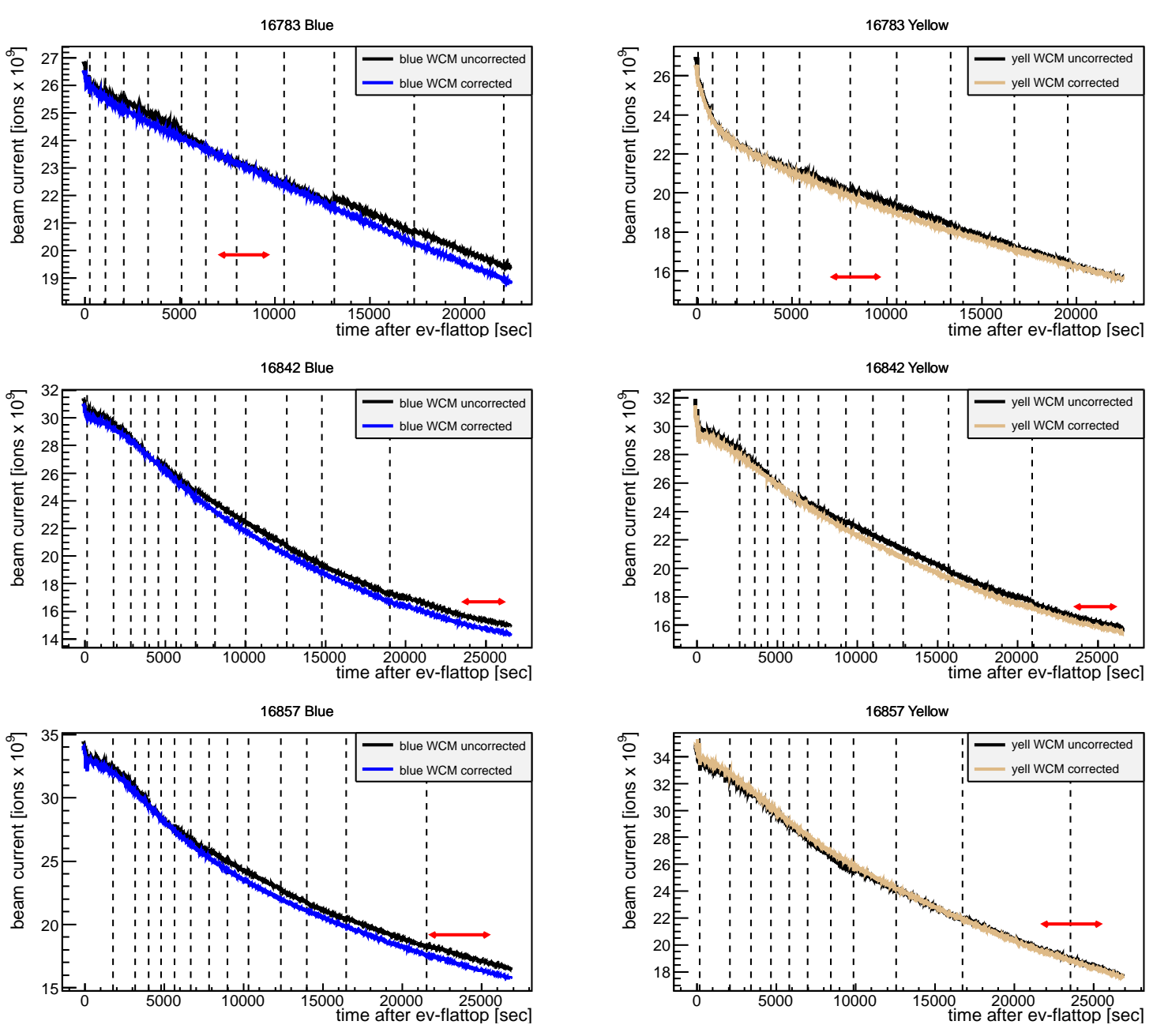

Figure 8: Corrected and uncorrected WCM readings as a function of time after "flattop" for the three vernier scan stores. The time of gain changes is indicated by vertical dashed lines.

WCM with and without corrections (as shown in Fig. 7) for the blue and yellow rings. Blue is shown on the left while yellow is shown on the right. All three stores with vernier scans are included, the times of the vernier scans are again added as red double arrows. The associated systematic error is based on the RMS of the blue shaded distribution shown in Fig. 6 and totals 1.2\% for the two beams together. 


\subsubsection{Fill Pattern}

In Eq. 3 an average beam intensity per bunch is used independent of the actual bunch pairs colliding at a given IP. Generally this is a very good approximation but the true value can deviate in case of very unevenly filled bunches. This is indeed the case for stores 16842 and 16857 in particular and cooled stores in general. Fig. 9 shows store 16842 as an example. The top graph shows the bunch intensity for both beams as a function of
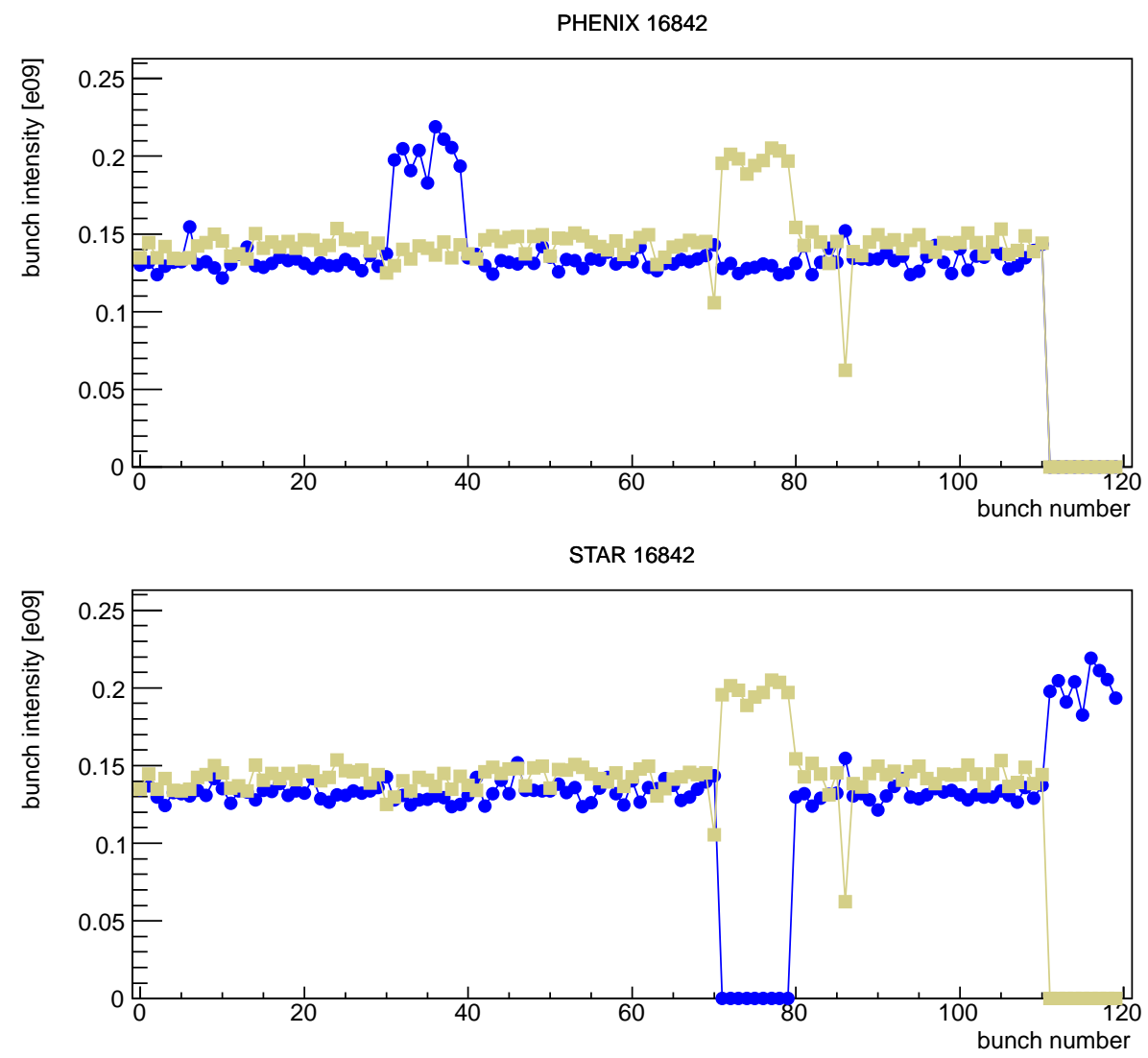

Figure 9: Bunch intensity as a function of yellow bunch number for the two beams according to colliding pairs in PHENIX (top) and STAR (bottom).

the yellow bunch number. In the PHENIX IP blue bunch \#1 collides with yellow bunch \#1 and the abort gaps line up. The bottom graph also shows the bunch intensity for both beams as a function of the yellow bunch number. In this graph, however, the blue bunch train is shifted by 60 degrees such that blue bunch \#41 collides with yellow bunch \#1. The abort gaps do not line up causing some yellow and some blue bunches to collide with the gap in the other beam. Due to the different levels of burn-of the bunches with only one collision clearly show a signficantly higher bunch intensity than the other quite evenly filled bunches. In this snapshot the intensity of one-collision bunches exceeds the one of bunches with two collisions by $30 \%$. This causes a significant correction to the term $\left(n_{B} n_{Y}\right) /\left(n_{\text {coll }} N_{B} N_{Y}\right)$ in Eq. 3 for IP6 in the two stores with cooled beams. In this analysis, average bunch currents and thus cross section and instantaneous luminosity measurements are corrected for this effect. Correction factors vary between $0.2 \%$ (IP8, 16783) up to $6.6 \%$ (IP6, 16842). No additional systematic uncertainty is assigned to this 
effect since it can be measured and corrected for.

\subsection{Beam Position Monitors}

Beam position monitors enter a discussion of vernier scan uncertainties in two ways:

- How accurate is the determination of the distance between the two beams during the vernier scan?

- Is there a crossing angle at the IPs reducing the maximum achievable luminosity?

For the first question only relative position measurements matter, i.e. how well the BPMs are capable to measure a deliberate beam displacement of a few hundred microns. The relative accuracy could be very good even at the presence of a large absolute position error. The quality of the relative measurement determines the accuracy of the width measurement in the vernier scan.

For the second question the accuracy of the absolute position measurements on either side of the IP matters. If significant true offsets of the beam position on both or just one side of the IP are present a virtual crossing angle could result as well as the opposite, i.e. a true crossing angle could go unnoticed due to false position measurements. Both types of measurement accuracies are discussed below.

\subsubsection{Relative Measurements}

During a vernier scan the wanted beam position (i.e. the set value for the scan request) as well as the measured beam position are recorded. Fig. 10 shows a typical instance of such a data set from the vernier scan in fill 16842. The Panel contains four graphs, each showing the measured beam position for the blue beam (top) and the yellow beam (bottom) as a function of the set value. The BPMs on the two sides of IP8 are shown individually. In this particular example the yellow beam was scanned across the blue. The consequent variation of the beam position in the blue ring indicates a sufficient beambeam interaction between the two beams to cause a small effect. The typical "S"-shape is not present for the first scan when beams were un-cooled and is less pronounced for the third. The resulting variation even in the shown case is small, no larger than $15 \mu \mathrm{m}$ peak to peak (and smaller than in the case of scanning $255 \mathrm{GeV}$ protons [6]). Nevertheless, it is taken into account when the distance between the two beams is calculated. Therefore no systematic error is dedicated to the beam-beam effect, instead it is corrected for and covered with the relative position measurement error.

For the shown yellow data set the deviation of the fitted slope from "1" is small, $3.5 \%$ and $+3 \%$ for $\mathrm{DxY} 7 \mathrm{H}$ and $\mathrm{DxY} 8 \mathrm{H}$ respectively, thus resulting in an agreement of the interpolated measured position at the IP to the set value of better than $1 \%$. The interpolated measured position at the IP, PosIP, is given by:

$$
\operatorname{Pos}_{\mathrm{IP}}=\frac{1}{2}\left(\mathrm{BPM}_{\mathrm{in}}+\mathrm{BPM}_{\text {out }}\right),
$$

with "in" and "out" referring to the two sides of the IP (in-coming and out-going). Tab. 4 summarizes the agreement of the measured position at the IP with the set values for both IPs and all three scans. The slopes all scatter around "1" (i.e. perfect agreement) by about $\pm 1 \%$ and show no consistent affinity towards an over- or under-shoot. The measured scatter includes beam-beam as well as potential hysteresis effects. Because of the excellent agreement actual beam positions measurements were used in this analysis 
16842 BluHor Sec. 7 BPM

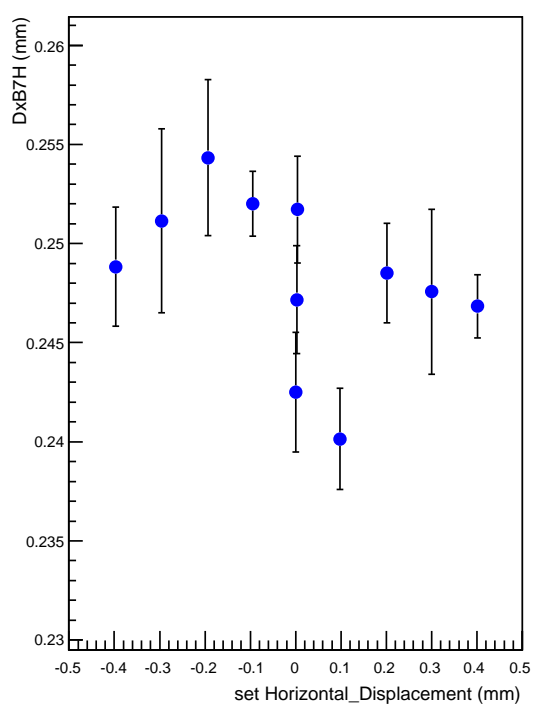

16842 YelHor Sec. 7 BPM

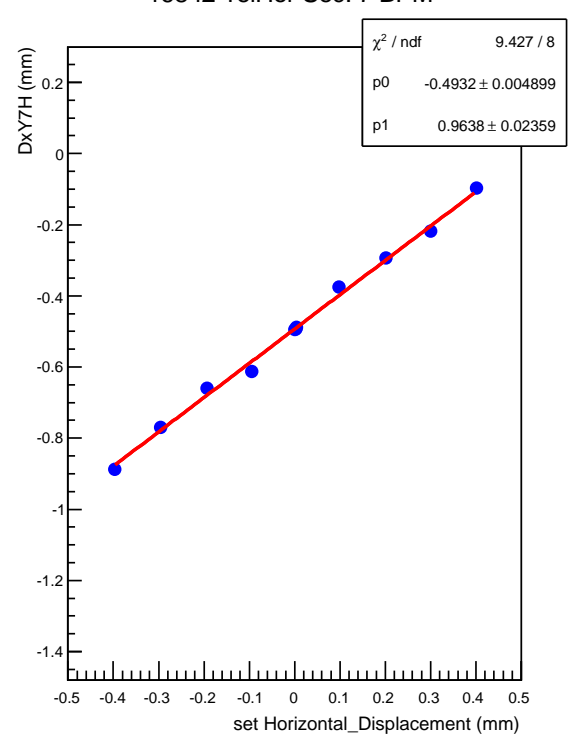

16842 BluHor Sec. 8 BPM

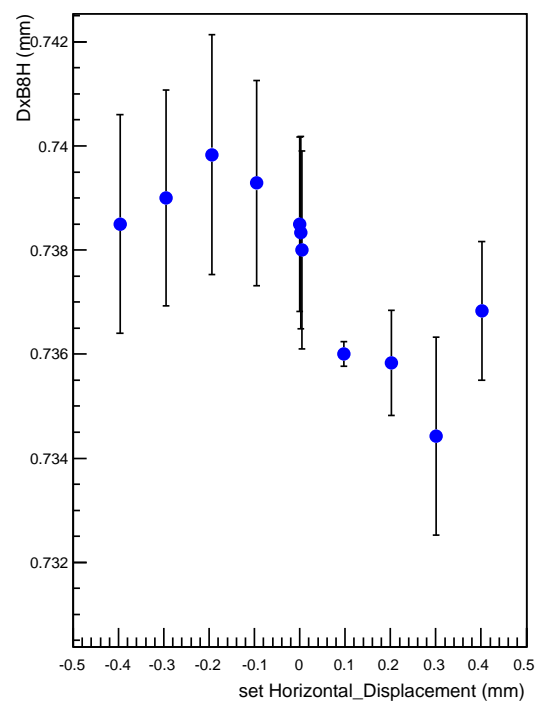

16842 YelHor Sec. 8 BPM

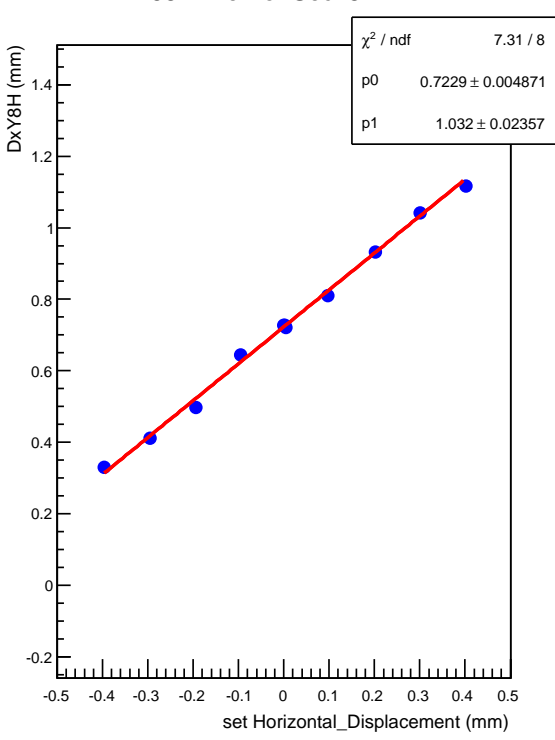

Figure 10: Beam position measurements during the horizontal part of the vernier scan in fill 16842 in IP8. All four horizontal DX BPMs are presented. Top row shows blue BPMs, the bottom row shows yellow BPMs.

(instead of the "set" values from the model) and a systematic error of $1.0 \%$ is assigned to the relative beam position measurement. No systematic error is dedicated to hysteresis specifically since it is covered by the above quoted error of $1 \%$. 


\begin{tabular}{|c|c|c|c|}
\hline & 16783 & 16842 & 16857 \\
\hline Hor IP6 & 1.006 & 0.99 & 0.99 \\
Ver IP6 & 0.99 & 1.0 & 1.00 \\
\hline Hor IP8 & 1.006 & 0.998 & 1.00 \\
Ver IP8 & 1.013 & 1.02 & 1.016 \\
\hline
\end{tabular}

Table 4: Slopes fitted to measured separation as a function of set separation. A slope of "1" corresponds to perfect agreement between the two.

\subsubsection{Absolute Measurement}

The differences between the blue and yellow beam positions in the two planes while beams are overlapping fully (i.e. after a successfull luminosity optimization such as a vernier scan) indicate the range of absolute beam position measurement errors since the expectation value is " 0 ". Fig. 11 shows the measured blue beam position at IP8 in the blue vertical plane ("blueVer"), the blue horizontal plane ("blueHor"), the yellow horizontal plane ("yellHor") and the yellow vertical plane ("yellVer") for fill 16850 as a function of the combined blue and yellow beam intensity ("AvgBeamCurrent"). The short-lived and

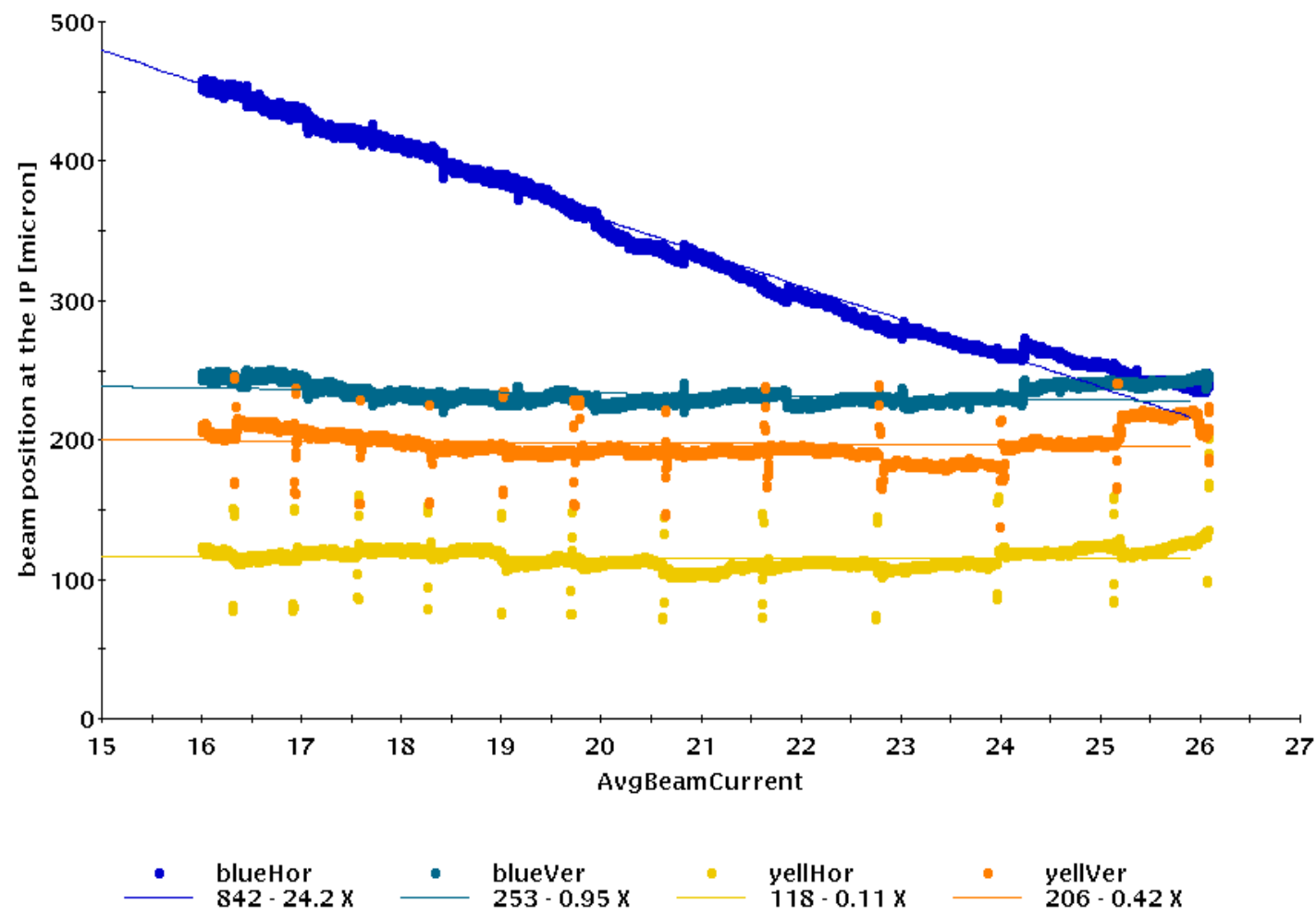

Figure 11: Horizontal and vertical blue beam position at the PHENIX IP measured by the DX BPM as a function of total beam current.

regular excursions apparent in the yellow data are a telltale of regular automatic luminosity optimizations. All four data sets are fitted with a $1^{\text {st }}$ order polynomial. While both vertical 
data sets as well as the yellow horizontal data set are all consistent with a constant, the blue horizontal dataset reveals an obvious dependency and increase of the measurement with a decreasing beam current. In this particular case it appears as if the beam drifted by 220 microns during the store which would, for cooled beams, correspond to more than $3 \sigma$ ! With such a separation, the instantaneous luminosity would have dropped to nothing provided that the other beam's position doesn't drift as well. Clearly, however, yellow horizontal is as constant as the two vertical planes. In addition, the regular automatic orbit corrections in combination with luminosity re-optimizations ought to keep the blue horizontal beam position stable and luminosity maximized. Therefore the drift, that can be traced back to the blue horizontal DX BPM on the sector 7 side of PHENIX (DxB7H), has to be fake.

Most stores (approx. 75\%) in the UU-run display this fake drift feature, with the yellow horizontal and vertical position as stable as the blue vertical one in this picture and the blue horizontal one drifting. Beam intensities in the UU run, with a typical bunch

\begin{tabular}{|c|r|r|r|c|c|}
\hline & 16783 & 16842 & 16857 & all stores & mean \\
\hline Hor IP6 & 20 & 40 & -10 & -30 to 80 & 30 \\
Ver IP6 & -20 & -30 & -20 & -80 to 10 & -40 \\
\hline Hor IP8 & 140 & 100 & 80 & 80 to 190 & 135 \\
Ver IP8 & 40 & 60 & 40 & 10 to 90 & 50 \\
\hline
\end{tabular}

Table 5: False beam separation measured by DX BPMs at the IPs after optimization in units of microns.

intensity at the beginning-of-store of $0.3 \times 10^{9}$, were small compared to $\mathrm{Au}$-beams with up to $1.3 \times 10^{9}$ ions/bunch in previous years. Thus I assume that the fake drift is not only correlated with but caused by the small and decreasing beam intensity although the measured drift slopes vary by more than a factor 2 from store to store and most DX BPMs seem not affected and not even $\mathrm{DxB} 7 \mathrm{H}$ demonstrates it in all stores. The described fake drift is present in all 3 vernier scan stores. Therefore, to estimate the measured difference between the beams I will use the position measurement with the highest beam intensity, i.e. the beginning-of-store after the first optimization. Tab. 5 summarizes the results. The mean measured beam separation varies between -40 micron and +135 micron depending on the plane and IP. At the same time we know the expected true value with luminosity optimized beam positions is 0 . The measurements scatter from store to store with \pm 50 microns around this mean. Picking the worst case, i.e. Hor IP8, yields 135 microns, an amount that could manifest itself on both sides of the IP. The corresponding uncertainty in terms of a crossing angle yields $0.015 \mathrm{mrad}$.

Fig. 12 shows the PHENIX ZDC coincidence rate in units of $\mathrm{kHz}$ as a function of the vertical crossing angle measured by the DX BPMs in IP8. A Gauss-fit of the data is superimposed. In case of a $15 \mu \mathrm{rad}$ crossing angle the achievable collision rate would be reduced by $1 \%$ from its maximum. In general, there could be a crossing angle present in both planes independently, doubling the effect. Thus the uncertainty due to unknown crossing angles totals $2 \%$. 


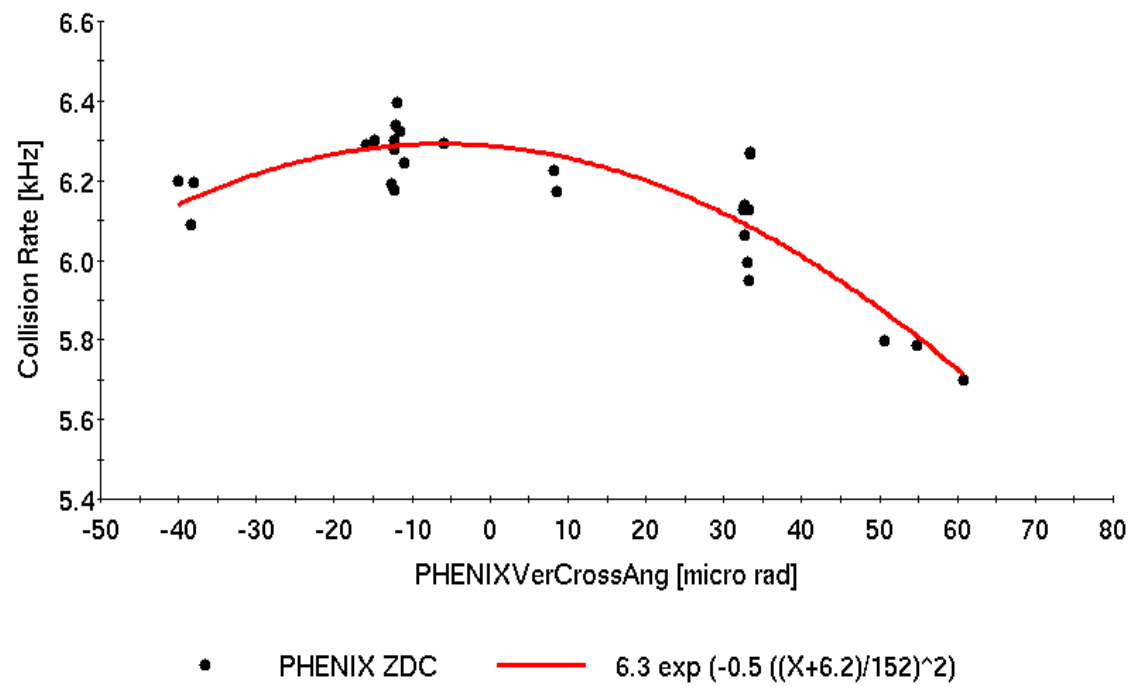

Figure 12: Vertical crossing angle scan in store 16857 in PHENIX. A Gauss-fit of the data is shown as well.

\section{Results}

After applying all corrections the effective ZDC cross sections and instantaneous luminosities for STAR and PHENIX are listed in Tab. 6. The three measurements per IP are all

\begin{tabular}{|l|c|c||c|c|}
\hline \multirow{2}{*}{ fill } & \multicolumn{2}{|c||}{ STAR } & \multicolumn{2}{c|}{ PHENIX } \\
\cline { 2 - 5 } & $\sigma_{\text {eff }}^{\text {ZDC }}[$ barn $]$ & $\mathcal{L}\left[10^{25} \mathrm{~cm}^{-2} \mathrm{~s}^{-1}\right]$ & $\sigma_{\text {eff }}^{\text {ZDC }}[$ barn $]$ & $\mathcal{L}\left[10^{25} \mathrm{~cm}^{-2} \mathrm{~s}^{-1}\right]$ \\
\hline 16783 & $15.70 \pm 0.47$ & $8.05 \pm 0.24$ & $16.09 \pm 0.48$ & $8.06 \pm 0.24$ \\
16842 & $16.05 \pm 0.48$ & $28.00 \pm 0.84$ & $15.66 \pm 0.47$ & $31.24 \pm 0.62$ \\
16857 & $15.78 \pm 0.47$ & $35.09 \pm 1.05$ & $15.65 \pm 0.47$ & $37.56 \pm 1.13$ \\
\hline
\end{tabular}

Table 6: Effective ZDC cross sections and instantaneous luminosities at the two IPs with statistical errors.

consistent with each other and with statistical scatter. The combined cross section measurements are shown in Fig. 13 and yield $15.84 \pm 0.27 \mathrm{~b}$ for STAR and $15.79 \pm 0.27 \mathrm{~b}$ for PHENIX. The effective cross sections of the two ZDCs are identical within the statistical errors.

Measured beam-overlap sizes, another result from vernier scans, are summarized in Tab. 7. From the fit to the vernier scan data it is impossible to distinguish between the blue and yellow beam therefore equal beamsizes were assumed when calculating the emittance. The emittance values are hour-glass corrected and listed in the right half of Tab. 7. The values in this table were all calculated using a model $\beta^{*}$ value of $0.7 \mathrm{~m}$ for the two rings and both planes. Results from the IPM [10] are added for comparison. The agreement is rather good with the exception of the horizontal data from store 16783 and 16857 where the IPM measurements are $25 \%$ and $20 \%$ below the results from the vernier scan. This discrepancy cannot be explained by wrong beta-functions being used since such 
UU $96 \mathrm{GeV}$ (run 12)

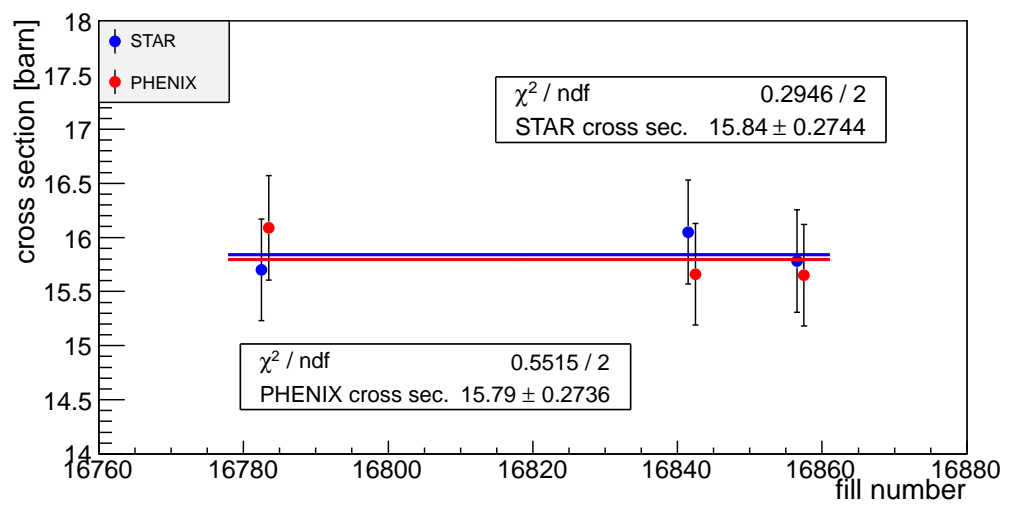

Figure 13: Cross sections as measured by the 3 vernier scans as a function of the fill number. All corrections applied.

\begin{tabular}{|l|c|c|c||c|c|c|}
\hline device & $\sigma^{\mathrm{vs}} 16783$ & $\sigma^{\mathrm{vs}} 16842$ & $\sigma^{\mathrm{vS}} 16857$ & $\epsilon^{\mathrm{RMS}} 16783$ & $\epsilon^{\mathrm{RMS}} 16842$ & $\epsilon^{\mathrm{RMS}} 16857$ \\
\hline Hor VS IP6 & $242 \pm 7$ & $95 \pm 3$ & $98 \pm 3$ & $2.9 \pm 0.1$ & $0.35 \pm 0.01$ & $0.38 \pm 0.01$ \\
Hor VS IP8 & $249 \pm 7$ & $94 \pm 3$ & $96 \pm 3$ & $3.0 \pm 0.1$ & $0.34 \pm 0.01$ & $0.36 \pm 0.01$ \\
Hor IPM & - & - & - & $2.2 \pm 0.2$ & $0.33 \pm 0.03$ & $0.3 \pm 0.03$ \\
\hline Ver VS IP6 & $242 \pm 7$ & $90 \pm 3$ & $96 \pm 3$ & $2.9 \pm 0.1$ & $0.31 \pm 0.01$ & $0.37 \pm 0.01$ \\
Ver VS IP8 & $249 \pm 7$ & $88 \pm 3$ & $93 \pm 3$ & $3.0 \pm 0.1$ & $0.30 \pm 0.01$ & $0.34 \pm 0.01$ \\
Ver IPM & - & - & - & $2.8 \pm 0.3$ & $0.3 \pm 0.03$ & $0.35 \pm 0.03$ \\
\hline
\end{tabular}

Table 7: Measured size of the beam overlap region in units of $\mu \mathrm{m}$ and calculated RMS normalized emittances in units of $\mathrm{mm}$ mrad. Model beta functions were used. Supplied errors are statistical only. IPM emittances for the two rings were combined into one value assuming errors of $10 \%$.

beta-beat would not change from store to store. There is no evidence of a deviation from the round beam assumption in the data, in fact the horizontal and vertical beam sizes are identical within statistical errors. There is also no evidence of the beam size being larger in one IP versus the other indicating equal beta functions in the two experimental IPs.

Nevertheless, the available data was compared with measured beta-functions from [11]. Measured and model beta functions are summarized in Tab. 8. According to the measured $\beta^{*}$ values at the minimum ( $1^{\text {st }}$ row in Tab. 8$)$ the beta function in the vertical plane exceeds the one in the horizontal plane by $15 \%$ in IP 6 and by $25 \%$ in IP 8 . In IP 8 the effect would be about twice as large as the statistical errors and should be visible in the vernier scan data. However, as stated above, there is no evidence for an unequality between the two planes, if anything the vertical beam size appears to be smaller than the horizontal one, not larger.

Taking into account the measured shift of the waist of the beta function away from the center of the interaction region $\left(2^{\text {nd }}\right.$ row in Tab. 8$)$, the beta-function disparity between the two planes becomes more pronounced, $20 \%$ of the horizontal $\beta^{*}$ in IP6 and $100 \%$ in IP8. Such a large disparity as in IP8 can be excluded with certainty. In addition to that, the measured-at-IP approach would result in a vertical beam size in IP8 that exceeds the 


\begin{tabular}{|l|c|c|c|c||c|c|c|c|}
\hline & \multicolumn{4}{|c||}{ STAR } & \multicolumn{4}{c|}{ PHENIX } \\
\cline { 2 - 9 } & BH & BV & YH & YV & BH & BV & YH & YV \\
\hline measured at min. & 0.64 & 0.93 & 0.75 & 0.63 & 0.76 & 0.99 & 0.61 & 0.70 \\
measured at IP & 0.65 & 1.06 & 0.82 & 0.66 & 0.82 & 1.97 & 0.63 & 0.70 \\
U12-v3 & 0.72 & 0.71 & 0.72 & 0.71 & 0.72 & 0.72 & 0.71 & 0.72 \\
\hline
\end{tabular}

Table 8: Model (U12-v3) and measured beta-functions (taken from [11]) at IP6 and IP8 in units of $[\mathrm{m}]$.

one in IP6 by 3 times the quoted statistical error. As before, there is no indication for such disparity in the data. Therefore, in this analysis, model beta-function values were assumed.

\subsection{Consistency Checks}

As a consistency check, the ratio of the instantaneous luminosities in STAR and PHENIX at the times of the three vernier scans are shown in Figure 14. The two vernier scans in store 16783 were done early in the store while the luminosity was still dropping more rapidly than at the end of a store. Therefore, the instantaneous luminosity of the later scan, the one in IP8, was scaled back to the time of the earlier scan, the one in IP6. This was done before the ratios were calculated. The average ratio yields 1.092. Due to the

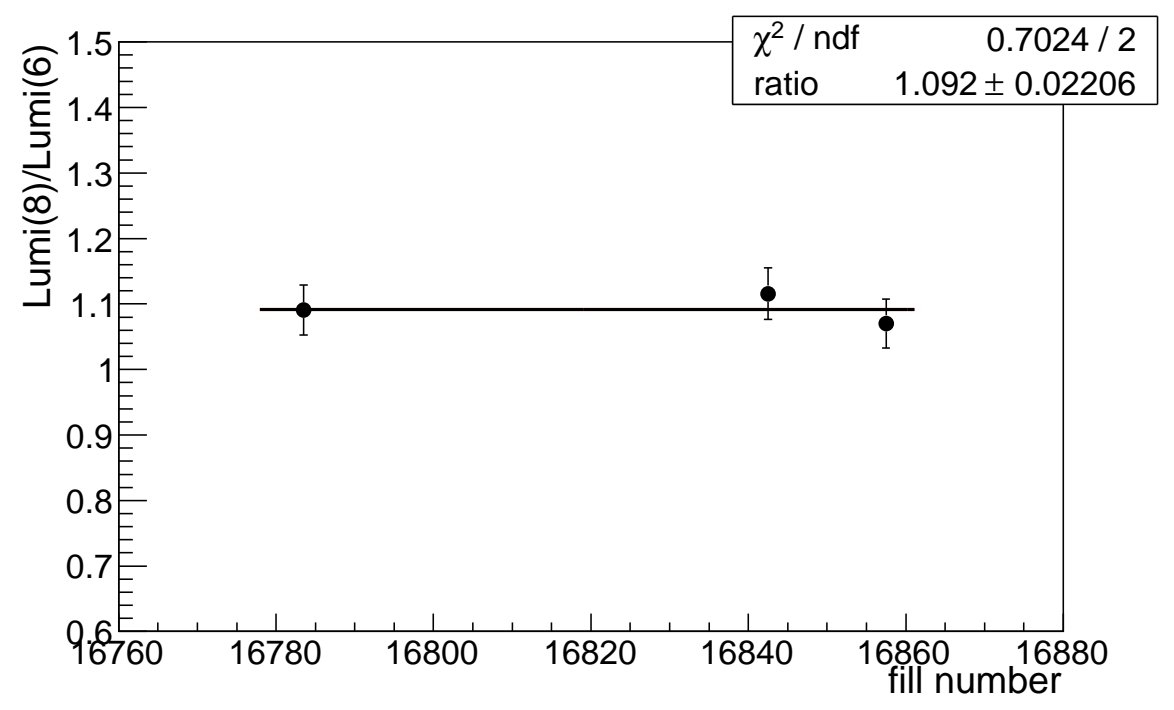

Figure 14: Ratio of instantaneous luminosities in PHENIX and STAR according to Tab. 6.

abort gap line-up in IP8 (compare section 4.1.4), the ratio of the expected instantaneous luminosities in PHENIX and STAR corresponds to $111 / 102=1.09$, the ratio of the total number of colliding pairs. The number derived from colliding pairs and the measured ratios are fully consistent with each other, no other effects seem to be at work. Keep in mind that in case of significantly different $\beta^{*}$-values in the two experiments the luminosity 
ratio would be expected to change accordingly. There is no evidence for unequal $\beta^{*}$-values. This is an additional argument to dismiss the measured $\beta^{*}$-values quoted in Tab. 8 even though the vernier scan method cannot help to determine the individual values themselves. Vernier scans are, however, quite sensitive to a disparity between the two IPs as well as the two planes.

The ratio of 111/102 as well as identical effective cross sections for the two experiments are further supported by our archived data. Fig. 15 shows the online coincidence
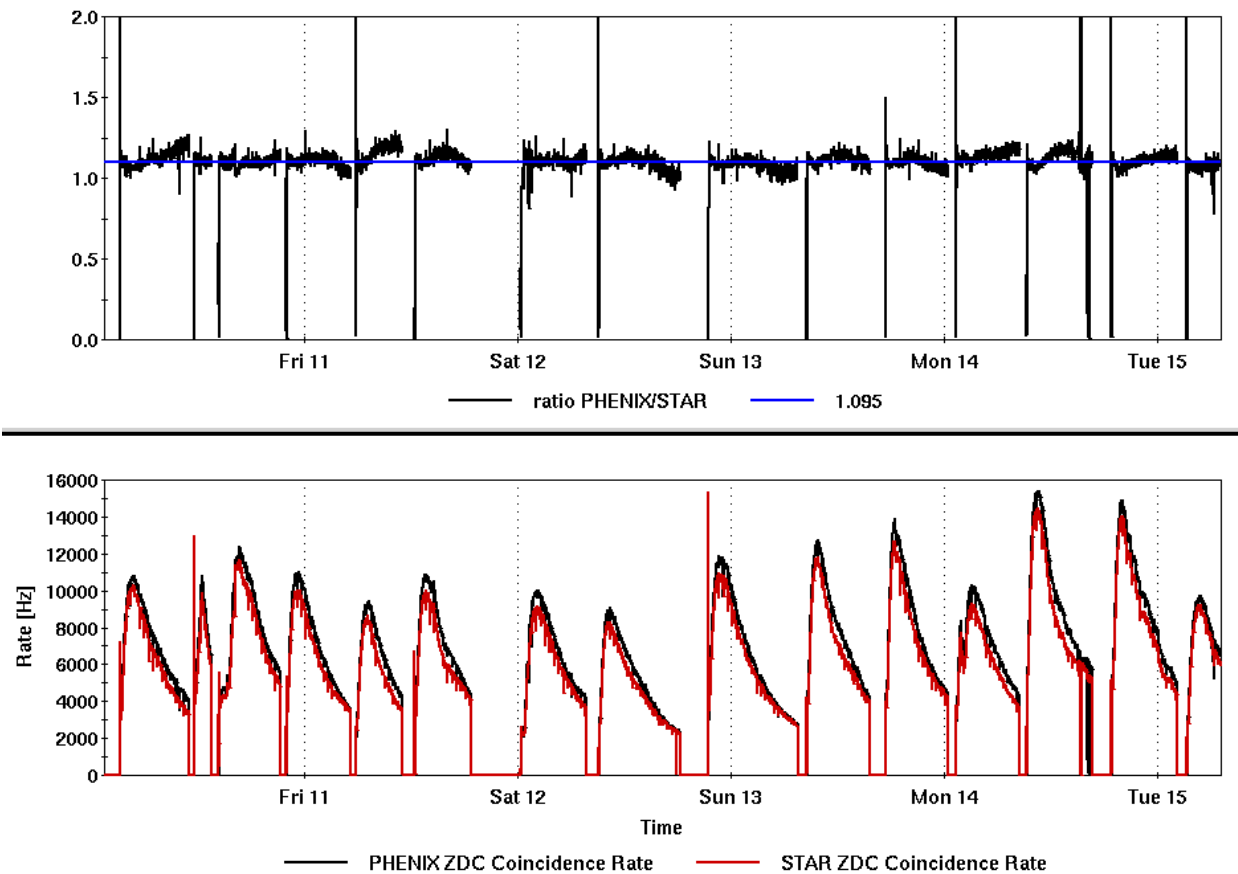

Figure 15: ZDC coincidence rates from the two experiments (bottom) and the ratio between the two (top) for the last 15 stores in the UU run.

signals from STAR and PHENIX (bottom) as well as the computed ratio between the two signals (top). Data from the last 15 stores of the UU run in 2012 are included. Regular automatic luminosity optimizations, done every 30 minutes, guaranteed optimal values for both experiments. The fitted average ratio for those 15 stores yields a convincing ratio of 1.095 between PHENIX and STAR luminosities. This is in excellent agreement with the expected value of 111/102 as well as the value from vernier scans provided model beta functions are used.

\section{Summary}

Combining the results, various systematic errors and the statistical error from above, this analysis adds up to the following effective cross sections:

- STAR: $15.84 \mathrm{~b} \pm 1.7 \%$ (stat.) $\pm 3.6 \%$ (sys.) 
- PHENIX: $15.79 \mathrm{~b} \pm 1.7 \%$ (stat.) $\pm 3.6 \%$ (sys.)

Note that the systematic error incorporates a total of $2.8 \%$ from beam current measurements, $1.0 \%$ from relative beam position measurements and $2 \%$ from absolute beam position measurements (crossing angles).

\section{Acknowledgements}

I thank my colleagues Christoph Montag, Mike Blaskiewicz and Wolfram Fischer for numerous fruitful and engaging discussions on this subject.

\section{References}

[1] S. Van Der Meer, ISR-PO/68-31, KEK68-64.

[2] M.A. Furman, M.S. Zisman "Luminosity", Handbook of Accelerator Physics and Engineering, Chapter 4, P. 247-270, 1999.

[3] J.M.Brennan, M.Blaskiewicz, K. Mernick "Stochastic Cooling in RHIC", IPAC12 proceedings, New Orleans, 2012.

M. Blaskiewicz, J. M. Brennan, K. Mernick, "RHIC Luminosity Increase with Bunched Beam Stochastic Cooling", COOL'13 proceedings, Murren 2013.

[4] K.A.Drees (BNL), S. White (CERN), "Vernier Scan Results from the First RHIC Proton Run at $250 \mathrm{GeV}$ ", IPAC10 Proceedings, 2010.

[5] A. Baltz et al., Nucl. Instr. and Methods, A417 (1998) 1

[6] A. Drees, "Analysis of Vernier Scans during RHIC Run-13", C-AD AP note C$\mathrm{A} / \mathrm{AP} / 488,2013$.

[7] A. Piwinski, "Touschek Effect and Intrabeam Scattering", Handbook of Accelerator Physics and Engineering, World Scientific 1999, p.125-127.

[8] http://www.cadops.bnl.gov/Instrumentation/InstWiki/index.php/ RHIC_Current_Transformer

[9] code "lineup.f", originally written by M. Blaskiewicz. Modified for the purposes of this analysis in 2013.

[10] R. Connolly et al., "RESIDUAL-GAS-IONIZATION BEAM PROFILE MONITORS IN RHIC", Proceedings of BIW10 Conference, Santa Fe, New Mexico, US, 2010.

[11] M.Bai, private communication 